\title{
Initial conditions for heavy ion collisions with QCD kinetic theory
}

\author{
Aleksi Kurkela \\ Theoretical Physics Department, CERN, Geneva, Switzerland \\ Faculty of Science and Technology, University of Stavanger, 4036 Stavanger, Norway \\ E-mail: a.k@eern.ch
}

\section{Aleksas Mazeliauskas*}

Institut für Theoretische Physik, Universität Heidelberg, Heidelberg 69120, Germany

E-mail: a.mazeliauskas@thphys.uni-heidelberg.de

\section{Jean-François Paquet}

Department of Physics, Duke University, Durham, NC 27708, USA

E-mail: jeanfrancois.paqueteduke.edu

\section{Sören Schlichting}

Department of Physics, University of Washington, Seattle, WA 98195-1560, USA

E-mail: sslng@uw. edu

\section{Derek Teaney}

Department of Physics and Astronomy, Stony Brook University, Stony Brook, NY 11794, USA

E-mail: derek.teaney@stonybrook . edu

\begin{abstract}
We employ the leading order QCD kinetic theory to describe a consistent matching between the initial stage of a heavy ion collision and the subsequent hydrodynamic evolution. We use the linearized kinetic response functions around the non-equilibirum longitudinally expanding background to map initial energy and momentum perturbations to the energy momentum tensor at hydrodynamic initialization time $\tau_{\text {hydro }}$. We check that hadronic observables then become rather insensitive to the cross-over time between kinetic theory and viscous hydrodynamics. The universal scaling of kinetic response in units of kinetic relaxation time $\tau_{R} \sim \eta /(s T)$ allows for a straightforward application of kinetic pre-equilibration event-by-event.
\end{abstract}

12th International Workshop on High-pT Physics in the RHIC/LHC Era

2-5 October, 2017

University of Bergen, Bergen, Norway

\footnotetext{
${ }^{*}$ Speaker.
} 


\section{Introduction}

The heavy ion collision is a multi-phase process spanning a wide range of energy scales. Many of experimental signatures are rather successfully reproduced by modern heavy ion models, which describe each stage and energy regime in a suitable theoretical approximation. In particular, the low energy expansion of Quark Gluon Plasma (QGP) is well described by relativistic viscous hydrodynamics $[1,2,3]$. The initial conditions for the hydrodynamic expansion are supplied by initial state models at hydrodynamic initialization time $\tau_{\text {hydro }}$. However most of initial state models do not contain the necessary physics to describe the approach to hydrodynamic behaviour and, therefore, create a mismatch of descriptions at the crossover time, which results in the dependence of physical observables on the auxiliary model parameter $\tau_{\text {hydro }}[6]$. A consistent theoretical overlap of initial stages and hydrodynamics could be important not only for low energy medium physics, like a build up of radial flow, but also for the high- $p_{T}$ physics of QGP, e.g. jets quenching and photon emission [7, 8].

In the high energy limit, the equilibration of weakly coupled QCD matter can be described by the leading order QCD kinetic theory $[9,10,11]$. We develop a framework of non-equilibrium kinetic response functions to smoothly map the initial conditions of far-from-equilibrium initial state models to hydrodynamically expanding matter and thus reduce the sensitivity to the hydrodynamic initialization time $\tau_{\text {hydro }}[12,13]$.

\section{Kinetic theory and universal scaling}

We use the leading order kinetic theory known as AMY formalism [9]. The QGP is then modelled by a collection of massless high energy quasi-particles (quarks and gluons) in a bath of soft momentum excitations. At leading order in the coupling constant $\lambda=N_{c} g^{2}$ the quasi-particles experience elastic $2 \leftrightarrow 2$ scatterings and inelastic $1 \leftrightarrow 2$ medium induced splittings. In a boost invariant expansion the QGP equilibrates according to the so called "bottom-up" thermalization scenario, which is supported by the first principles classical-statistical Yang-Mills simulations [10, $14,15]$. We would like to emphasize that the same kinetic framework can be also used to describe the energy loss of high- $p_{T}$ partons, and the electromagnetic emission in QGP [7, 8].

In this work we solve the Boltzman equation for the color averaged gluon distribution function $f(\tau, \mathbf{p}, \mathbf{x})$

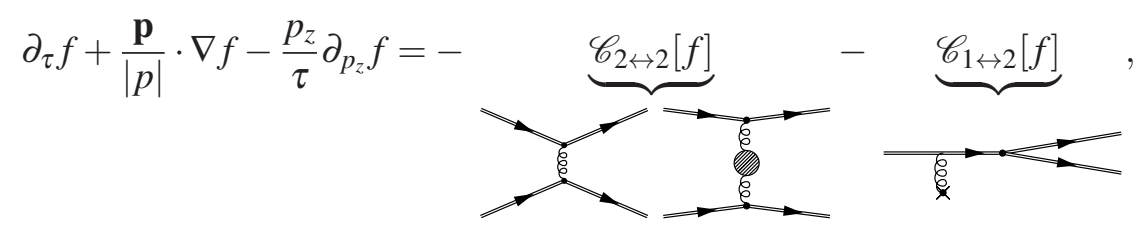

but neglect the effect of quark degrees of freedom ${ }^{1}$. The distribution function $f(\tau, \mathbf{p}, \mathbf{x})$ is decomposed into a spatially homogeneous, but anisotropic $\left\langle p_{\perp}^{2}\right\rangle \gg\left\langle p_{z}^{2}\right\rangle$ background $\bar{f}(\tau, \mathbf{p})$ and linearized fixed wavenumber $\mathbf{k}$ perturbations $\delta f(\tau, \mathbf{p}) e^{i \mathbf{k} \cdot \mathbf{x}}$ in the transverse plane of the collision. The resulting system of coupled equations is then solved [12].

\footnotetext{
${ }^{1}$ At early times the gluon occupation far exceeds that of quarks due to Pauli exclusion principle.
} 


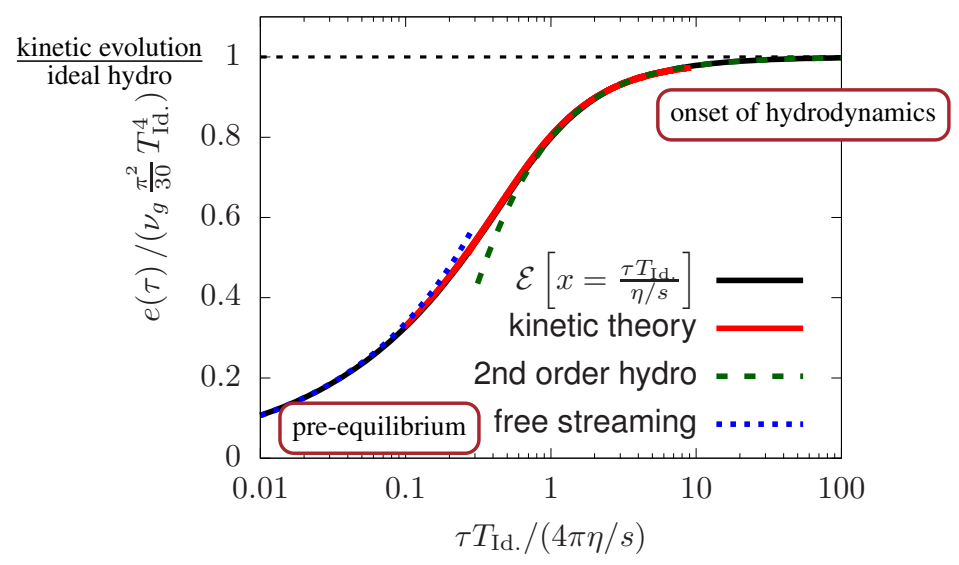

Figure 1: The universal scaling curve, Eq. (2.3), for energy density. Note that the kinetic theory curve corresponds to an overlapping kinetic evolution for a range of couplings $\lambda=10-25$, which correspond to $\eta / s=0.62-0.16$.

The key observation is that, as the system approaches equilibrium, many of the details of the initial distribution function is lost and only the first moments of the distribution function, i.e. energy momentum tensor, carries the necessary information for the subsequent hydrodynamic evolution. Furthermore at late times, the approach to hydrodynamics is approximately universal if measured in units of kinetic relaxation time $\tau_{R} \sim \eta /(s T)$. For example the energy evolution of a uniform Bjorken expanding background can be described by an asymptotic expansion in second order hydrodynamics [16]

$$
\text { scaling in hydro: } \quad e(\tau)=\underbrace{v_{g} \frac{\pi^{2}}{30} T_{\text {Id. }}^{4}}_{\text {"ideal" temp. }}(\underbrace{1}_{\text {ideal }}-\underbrace{\frac{8}{3} \frac{\eta / s}{\tau T_{\text {Id }}}}_{\text {viscous }}+\underbrace{C_{2}\left(\frac{\eta / s}{\tau T_{\text {Id. }}}\right)^{2}}_{\text {2nd order hydro }})
$$

where $T_{\text {Id. }}$ characterises the asymptotic temperature scale and $C_{2}$ is a second order transport coefficient with only weak dependence on the coupling constant $\lambda$. In practice, we find that for a range of moderate values of $\lambda$, the entire kinetic pre-equilibrium exhbits scaling and we define a scaling function $\mathscr{E}(x)$

$$
\text { generalized scaling: } \quad e(\tau)=v_{g} \frac{\pi^{2}}{30} T_{\text {Id. }}^{4}(\tau) \times \underbrace{\mathscr{E}\left[x=\frac{\tau T_{\mathrm{Id} .}(\tau)}{\eta / s}\right]}_{\text {non-equilibrium evolution }},
$$

which smoothly interpolates between the early time free-streaming like behavior and the late time hydrodynamics, as shown in Fig. 1. For homogeneous boost invariant background, the energy density function $e(\tau)$ completely determines the entire background energy momentum tensor $\bar{T}^{\mu v}=\operatorname{diag}\left(e, P_{T}, P_{T}, \frac{1}{\tau^{2}} P_{L}\right)$, where $P_{L}=-\partial_{\tau}(\tau e(\tau))$ and $e+2 P_{T}+P_{L}=0$.

\section{Linear response functions}

For central heavy ion collisions, e.g. PbPb, the physical description of initial stages $\tau \lesssim 2 \mathrm{fm}$ can be considerably simplified by using the approximate boost invariance symmetry and the fact 
that the transverse size of the collision is much larger than the duration of the pre-equilibrium stage, i.e. $R_{\mathrm{Pb}} \gg \Delta \tau$. Then the energy momentum tensor at a given point in the transverse plane $\mathbf{x}_{0}$ is completely determined by a small neighbourhood of points which lie in the causal past of the said point, i.e. $\left|\mathbf{x}-\mathbf{x}_{0}\right|<\Delta \tau$. Furthermore, the energy momentum tensor $T^{\mu v}$ in the causal neighbourhood can be decomposed into a homogeneous background $\bar{T}^{\mu v}$ and perturbations $\delta T^{\mu v}$. At linear order the evolution of such perturbations is determined by linear response functions, which, in Fourier space, is a linear map between initial energy and momentum perturbations and the final time energy momentum tensor ${ }^{2}$

$$
\underbrace{\frac{\delta T^{\mu v}(\tau, \mathbf{k})}{e(\tau)}}_{\text {output for hydro }}=\underbrace{\tilde{G}_{(\tau, i)}^{\mu v}\left(\mathbf{k}, \tau, \tau_{0}\right) \times}_{\text {non-equilibrium kinetic response }}[\underbrace{\frac{\delta T^{\tau \tau}\left(\tau_{0}, \mathbf{k}\right)}{e\left(\tau_{0}\right)} \text { or } \frac{\delta T^{\tau i}\left(\tau_{0}, \mathbf{k}\right)}{e\left(\tau_{0}\right)}}_{\text {initial perturbations }}]
$$

Such k-space Green functions are obtained from the moments of the distribution function of the linearised perturbations $\delta f(\tau, \mathbf{p}) e^{i \mathbf{k x}}$, which are evolved according to the coupled system of equations discussed in Sec. 2. Notably, the linear response functions become approximately invariant if written in terms of scaling variables $\tau T_{\text {Id. }} /(\eta / s)$ and $|\mathbf{k}|\left(\tau-\tau_{0}\right)$. This allows performing a computational intensive kinetic theory evolution for a particular value of $\lambda$ once, and then applying the scalable response functions to different values of effective $\eta / s$, time $\tau$ or temperature scale $T_{\text {Id. }}$.

\section{Results}

We apply the kinetic pre-equilibration stage to a central $\mathrm{PbPb}$ event with a centre-of-mass energy $\sqrt{s_{N N}}=2.76 \mathrm{TeV}$ and with IP-Glasma initial conditions. IP-Glasma is a successful initial state model motivated by Color Glass Condensate picture and includes a 2D Yang-Mills evolution of initial gluonic fields $[17,18]$. However, the classical Yang-Mills evolution does not lead to hydrodynamization, which is achieved by first passing the initial energy and momentum densities to a kinetic pre-equilibrium stage and then smoothly matching to viscous hydrodynamics at a later time $\tau_{\text {hydro. }}$. We check by varying the crossover time $\tau_{\text {hydro }}$ that the integrated and differential hydrodynamic fields, e.g. entropy density and radial velocity, are only weakly dependent on the cross-over time ${ }^{3}$. Finally, we extract the hadronic observables from the constant temperature freeze-out surface. For simplicity we only consider thermal spectra at the moment of freeze-out, i.e. neglecting resonance decays. In Fig. 2 we present the thermal pion multiplicity $d N_{\pi} / d y$, mean pion transverse momentum $\left\langle p_{T}^{\pi}\right\rangle$ and the elliptical flow $v_{2}^{\pi}$ for our event. We observe that the dependence of hadronic observables on the duration of kinetic pre-equilibrium stage is weak, therefore the hydrodynamic simulations of heavy ion collisions with kinetic pre-equilibrium stage could perhaps yield more reliable extractions of transport properties of QGP.

In summary, we presented a well motivated pre-equilibrium model based on a QCD kinetic theory with a smooth and consistent approach to hydrodynamics. Thanks to an approximate scaling

\footnotetext{
${ }^{2}$ In general one can also consider response to the spatial perturbations of energy momentum tensor, but here we focus only on the perturbations sourced by initial fluctuations of conserved charges of energy and momentum.

${ }^{3}$ One of the remaining shortfalls of the matching is the difference between the conformal equation of state of massless kinetic theory and a non-conformal lattice equation of state used in modern hydrodynamic codes.
} 

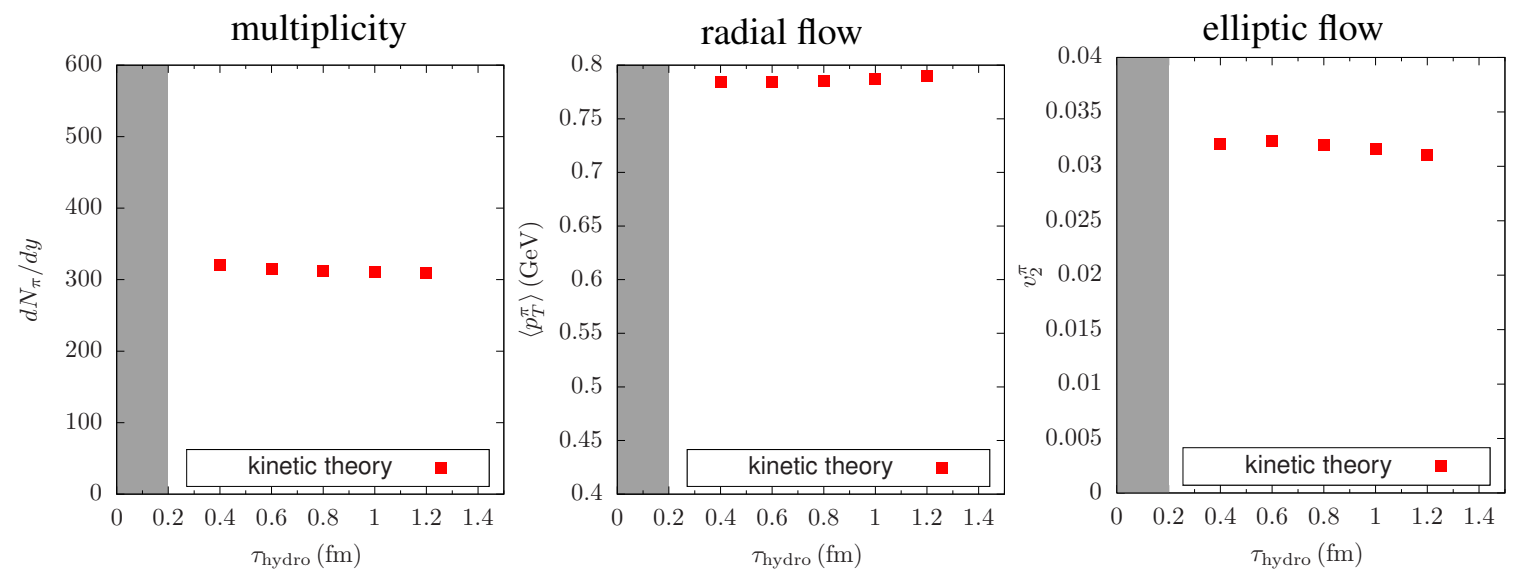

Figure 2: The final hadronic observables as a function of hydrodynamic initialization time $\tau_{\text {hydro }}$ with kinetic pre-equilibrium stage and IP-Glasma initial conditions for a $\sqrt{s}=2.76 \mathrm{Tev}$ central $\mathrm{PbPb}$ event. Different panels correspond to thermal pion multiplicity $d N_{\pi} / d y$, the mean transverse momentum $\left\langle p_{T}^{\pi}\right\rangle$ and the elliptic flow $v_{2}^{\pi}$.

with $\eta /(s T)$, the tabulated linear response functions can be straightforwardly applied to event-byevent initial conditions of heavy ion collisions and thus reduce the dependence on the auxiliary parameters of a hydrodynamic model of heavy ion collisions.

\section{Acknowledgments}

This work was supported in part by the DFG Collaborative Research Centre SFB 1225 (ISOQUANT) (A.M.).

\section{References}

[1] U. Heinz and R. Snellings, "Collective flow and viscosity in relativistic heavy-ion collisions," Ann. Rev. Nucl. Part. Sci. 63 (2013) 123 [arXiv:1301.2826 [nucl-th]].

[2] M. Luzum and H. Petersen, "Initial State Fluctuations and Final State Correlations in Relativistic Heavy-Ion Collisions,” J. Phys. G 41 (2014) 063102 [arXiv:1312.5503 [nucl-th]].

[3] D. A. Teaney, "Viscous Hydrodynamics and the Quark Gluon Plasma," arXiv:0905.2433 [nucl-th].

[4] W. van der Schee, P. Romatschke and S. Pratt, "Fully Dynamical Simulation of Central Nuclear Collisions," Phys. Rev. Lett. 111 (2013) no.22, 222302 [arXiv:1307.2539 [nucl-th]].

[5] P. Romatschke, "Light-Heavy Ion Collisions: A window into pre-equilibrium QCD dynamics?," Eur. Phys. J. C 75 (2015) no.7, 305 [arXiv:1502.04745 [nucl-th]].

[6] A. Kurkela, "Initial state of Heavy-Ion Collisions: Isotropization and thermalization," Nucl. Phys. A 956 (2016) 136 [arXiv:1601.03283 [hep-ph]].

[7] S. A. Bass, C. Gale, A. Majumder, C. Nonaka, G. Y. Qin, T. Renk and J. Ruppert, "Systematic Comparison of Jet Energy-Loss Schemes in a realistic hydrodynamic medium,” Phys. Rev. C 79 (2009) 024901 [arXiv:0808.0908 [nucl-th]]. 
[8] J. F. Paquet, C. Shen, G. S. Denicol, M. Luzum, B. Schenke, S. Jeon and C. Gale, "Production of photons in relativistic heavy-ion collisions," Phys. Rev. C 93 (2016) no.4, 044906 [arXiv:1509.06738 [hep-ph]].

[9] P. B. Arnold, G. D. Moore and L. G. Yaffe, "Effective kinetic theory for high temperature gauge theories," JHEP 0301 (2003) 030 [hep-ph/0209353].

[10] R. Baier, A. H. Mueller, D. Schiff and D. T. Son, “'Bottom up' thermalization in heavy ion collisions," Phys. Lett. B 502 (2001) 51 [hep-ph/0009237].

[11] A. Kurkela and Y. Zhu, "Isotropization and hydrodynamization in weakly coupled heavy-ion collisions," Phys. Rev. Lett. 115 (2015) no.18, 182301 [arXiv:1506.06647 [hep-ph]].

[12] L. Keegan, A. Kurkela, A. Mazeliauskas and D. Teaney, "Initial conditions for hydrodynamics from weakly coupled pre-equilibrium evolution,” JHEP 1608 (2016) 171 [arXiv:1605.04287 [hep-ph]].

[13] A. Kurkela, A. Mazeliauskas, J. F. Paquet, S. Schlichting and D. Teaney, in preparation.

[14] J. Berges, K. Boguslavski, S. Schlichting and R. Venugopalan, "Universal attractor in a highly occupied non-Abelian plasma,” Phys. Rev. D 89 (2014) no.11, 114007 [arXiv:1311.3005 [hep-ph]].

[15] J. Berges, K. Boguslavski, S. Schlichting and R. Venugopalan, "Turbulent thermalization process in heavy-ion collisions at ultrarelativistic energies,” Phys. Rev. D 89 (2014) no.7, 074011 [arXiv:1303.5650 [hep-ph]].

[16] R. Baier, P. Romatschke, D. T. Son, A. O. Starinets and M. A. Stephanov, "Relativistic viscous hydrodynamics, conformal invariance, and holography,” JHEP 0804 (2008) 100 [arXiv:0712.2451 [hep-th]].

[17] B. Schenke, P. Tribedy and R. Venugopalan, "Fluctuating Glasma initial conditions and flow in heavy ion collisions," Phys. Rev. Lett. 108, 252301 (2012) [arXiv:1202.6646 [nucl-th]].

[18] B. Schenke, P. Tribedy and R. Venugopalan, "Event-by-event gluon multiplicity, energy density, and eccentricities in ultrarelativistic heavy-ion collisions," Phys. Rev. C 86, 034908 (2012)

[arXiv:1206.6805 [hep-ph]]. 